\title{
Could multiresponsive hollow shell-shell nanocontainers offer an improved strategy for drug delivery?
}

\author{
"In this editorial, we report a new concept using \\ responsive nanocontainers for drug delivery, namely \\ employing hollow nanogels with two polymer shells that \\ have different volume phase transition temperatures."
}

First draft submitted: 5 September 2016; Accepted for publication: 9 September 2016; Published online: 26 October 2016

Keywords: drug delivery $\bullet$ hollow nanoparticles $\bullet$ nanogels $\bullet$ nanomedicine $\bullet$ uptake-release

In this editorial, we report a new concept using responsive nanocontainers for drug delivery, namely employing hollow nanogels with two polymer shells that have different volume phase transition temperatures. The temperature responsive swelling and collapse of the inner shell control the uptake, storage in the void and release of guest (macro) molecules, while the swelling of the outer shell controls the size of the void and the colloidal stability of the nanogels. In other words, the inner shell plays a role of a membrane of variable permeability for the molecules while the outer shell always serves as permeable membrane. These new multishell nanoparticles can be further functionalized. Their different layers may fulfill distinct requirements during transport, uptake and targeted release of pharmaceutical molecules. Therefore, two-shell hollow nanocontainers may have great potential to be used as drug delivery carriers for different applications.

Areas of interest for nanoparticle development are improved local drug release in diseased tissue, intracellular delivery of therapeutic nucleotide acids and improvement of oral bioavailability of large molecule therapeutics. Drugs for the treatment of malignant, infectious or autoimmune diseases such as chemotherapeutic drugs, antibiotics or immunosuppressors can have severe side effects due to toxicity, individual intolerances or provocation of infectious complications because its systemic effect on the immune system. One of the major problems is the systemic release of the drugs despite most diseases being localized to specific organs or areas of the body. Drug carriers for targeted release can specifically increase drug concentrations within the diseased tissue or cells leading to improved treatment efficacy and less adverse drug effects. Therapeutic DNA or RNA that directly influences gene expression within diseased cells requires specific vectors for intracellular delivery. Nanoparticles offer a great alternative to viral or bacterial vector systems. Several antibiotics and protein drugs such as insulin or therapeutic antibodies (biologicals) have to be administered by the parenteral route. Nanoparticle systems that improve the oral bioavailability of such drugs would facilitate the treatment in an out-patient setting. Thus, the development of nanocontainers, which allow for the uptake, storage and triggered release of functional agents, is one of the key challenges in nanomedicine.

\section{The advantage of multifunctional nanocarriers}

There are various tasks that nanocarriers have to fulfill in order to improve oral bioavailabilty and targeted release. First, drugs need to be protected from the harsh and
Walter Richtering

Author for correspondence: Institute of Physical Chemistry, RWTH

Aachen University, Landoltweg 2, 52056 Aachen, Germany richtering@rwth-aachen.de

Igor I Potemkin

Author for correspondence:

Physics Department, Lomonosov Moscow State University, 119991 Moscow, Russian Federation and

DWI-Leibniz Institute for Interactive Materials e.V., 52056 Aachen, Germany igor@polly.phys.msu.ru

\section{Andrey A Rudov}

Physics Department, Lomonosov Moscow State University, 119991 Moscow, Russian Federation and

DWI-Leibniz Institute for Interactive Materials e.V., 52056 Aachen, Germany

\section{Gernot Sellge}

Department of Internal Medicine III, University Hospital RWTH Aachen, Pauwelsstr. 30, 52074 Aachen, Germany

\section{Christian Trautwein}

Department of Internal Medicine III, University Hospital RWTH Aachen,

Pauwelsstr. 30, 52074 Aachen, Germany

\section{Future : Medicine part of}


diverse environment during the passage of the GI tract (e.g., $\mathrm{pH}$ between 2 and 10, digestive enzymes, different salt concentrations). Second, drugs have to cross several barriers with very different behavior such as the intestinal mucosa consisting of a mucus layer and a tight cellular epithelial monolayer, the endothelium of the blood vessels to reach and exit the systemic blood circulation and cellular membranes for drugs with an intracellular mode of action. Third, nanoparticles must confer colloidal stability within the blood in order to allow sufficient transport and prevention of embolic complications in small vessels. Fourth, nanoparticles should preferentially enrich in diseased tissue or cells. Fifth, the drug cargo should be preferentially released in diseased tissue or cells.

"...microgels have the ability to adapt their size and shape to environmental requirements and enable controlled size selective mass transfer."

Specific physicochemical conditions at organ barriers, cellular surfaces and within diseased tissue such as differences in $\mathrm{pH}$, salt concentration, charge, redox potential, protease activity and different pore sizes as well as external stimuli such as light, heat and cold can be exploited as triggers for nanocarrier uptake and cargo release $[1,2]$. Furthermore, coating of nanoparticle surfaces with specific ligands enhances interaction with cellular membranes or extracellular matrices and therefore facilitates targeted barrier crossing and cellular uptake [3].

Nanocarriers based on stimuli-responsive polymers in different architectures as, for example, dendrimers, micelles and nanohydrogels, and employing a variety of stimuli for the release as, for example, temperature, light, $\mathrm{pH}$, ultrasound or magnetic field have been developed [4-11]. The development of drug carriers that guarantee the stability of the drug and, in addition, respond to multiple stimuli may largely improve targeted drug release [3]. An example for dual-functional drug carriers is provided by Mao et al. [12]. They developed dual $\mathrm{pH}$-responsive polymeric micelles for drug release. At weakly acidic environment at $\mathrm{pH}$ approximately $6.5 \%$ in tumor environment (physiological $\mathrm{pH}$ is 7.4) the surface charge of the micelles reversed from negative to positive facilitating binding to positively charged cellular membranes and subsequent cellular uptake. At $\mathrm{pH}<5.5$ present in intracellular phagolysosomes the drug is released owing to the cleavage of imine bonds. This dual $\mathrm{pH}$-responsive feature may improve the ability to precisely deliver chemotherapeutic drugs to cancer cells [12]. Shan et al. reported self-assembled nanoparticles for efficient oral delivery of insulin. The nanoparticles possessed a nanocomplex core composed of insulin and cell-penetrating pep- tide, and a dissociable hydrophilic coating of poly $(\mathrm{N}-$ (2-hydroxypropyl)methacrylamide) copolymer derivatives. Nanoparticles exhibited excellent permeation in mucus due to the 'mucus-inert' poly(N-(2-hydroxypropyl)methacrylamide) copolymer coating, as well as high epithelial absorption mediated by cell-penetrating peptide [13].

Temperature-responsive nanoparticles may be used for targeted drug release in combination with hyperthermia therapy. Radiofrequency ablation (RFA) directly induces cell death by high frequency radio waves that are applied by a needle electrode into skin or liver tumors. Mild local hyperthermia applied by microwaves, high frequency ultrasound or infusion of warmed liquids does not induce cell death by itself but renders tumor cells more responsive to radiation or chemotherapy. Temperature-responsive nanocarriers for local release of chemotherapeutic drugs may improve RFA efficiency by improving killing of tumors cells not immediately killed by RFA alone [14] and increase local drug concentration in other forms of hyperthermia therapy [2].

\section{Multiresponsive hollow shell-shell nanocontainers}

Nanogels based on responsive polymers provide characteristic features that make them characteristically different from other colloidal and polymeric systems. The combination of being soft and porous while still having a stable structure through the cross-linked network allows for the possibility to introduce orthogonal chemical functionality in different compartments within the microgel $[15,16]$. In contrast to rigid colloidal nanoparticles, microgels have the ability to adapt their size and shape to environmental requirements and enable controlled size selective mass transfer [17]. Thus nanogels are soft species and can achieve switchable properties that enable adaptability of form and function both in bulk solution and at interfaces [18-20].

Hollow capsules represent a very promising structure for the design of efficient drug delivery systems $[4,21]$. Drugs could be stored in the void of the hollow particles with the shell protecting them from external influences but also a controlled release of the drugs by external stimuli is possible $[22,23]$. Hollow nanogels allow combining the typical features of capsules having a void with the adaptability of soft nanogels [24]. Recently we introduced hollow nanogels with two shells of different sensitivity to external stimuli [25]. These two-shell hollow nanocontainers are shown in Figure 1. The images are snapshots of the nanogels obtained at different conditions in computer simulations [25]. What makes the nanogels special and why could they offer a new strategy for nanomedicine? 


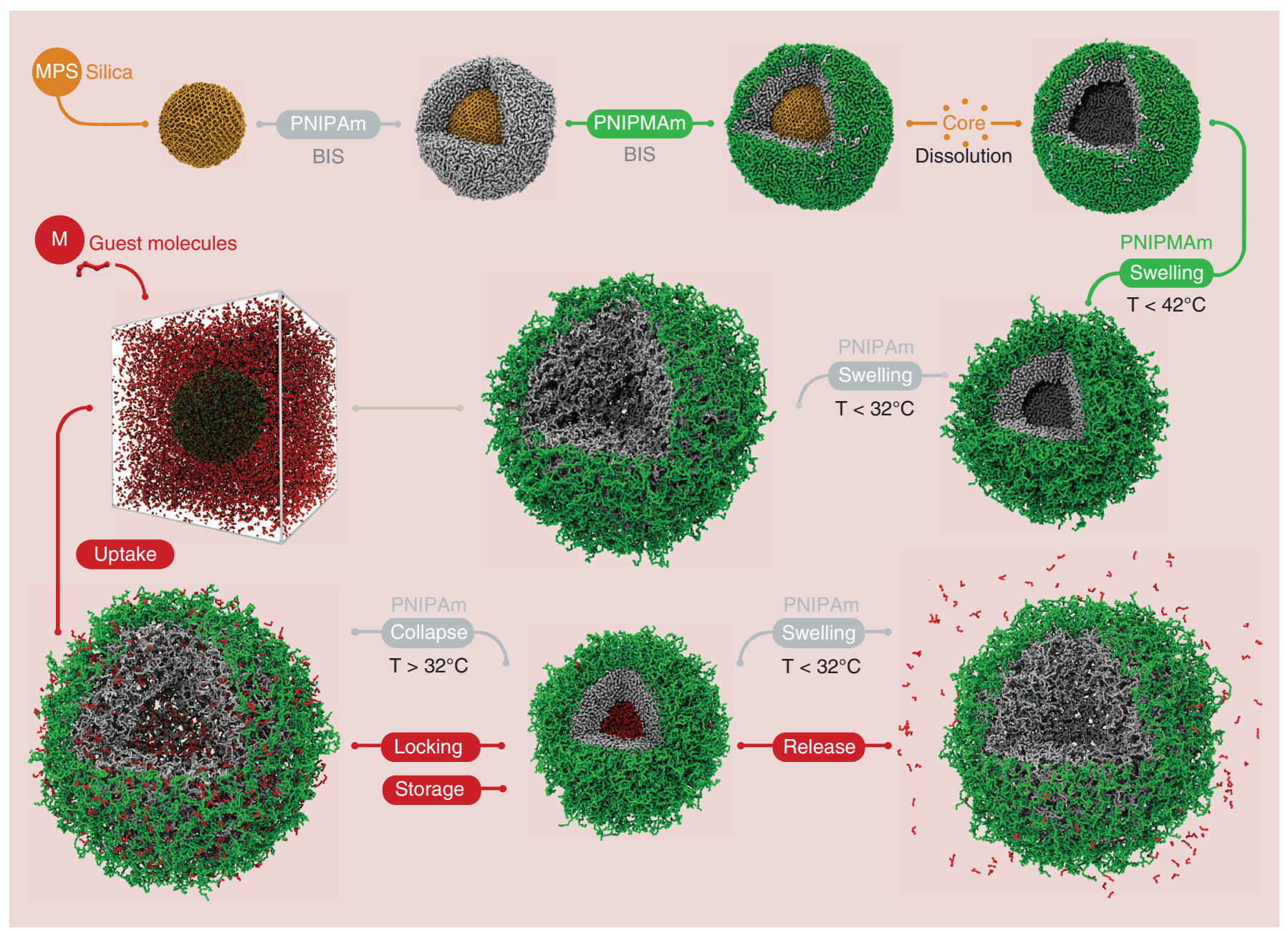

Figure 1. Snapshots of nanogels at different conditions obtained via computer simulations. (Upper row, left-to-right) Procedure of preparation of the hollow double-shell nanogels. (Middle row, right-to-left) Collapse $\left(T<42^{\circ} \mathrm{C}\right)$ and swelling $\left(\mathrm{T}<32^{\circ} \mathrm{C}\right)$ of the inner shell of the nanogel. Immersing of the nanogel with open pores into solution of the (guest) molecules (red dots). (Bottom row, leftto-right) Penetration of the molecules into the nanogel in the solution. Locking of the guest molecules in the void via collapse of the inner shell at $\mathrm{T}>32^{\circ} \mathrm{C}$. Release of the guest molecules into the pure solvent at $\mathrm{T}<32^{\circ} \mathrm{C}$ via swelling of the inner shell.

First of all, the 'membrane' consists of two polymer networks that are chemically connected but consist of different polymers. On the one hand this allows separating different relevant functions of the membrane: the responsive swelling of the inner network allow controlling the permeability of the (inner) shell, whereas the swelling of the outer shell provides the colloidal stability. The synthesis of the two-shell nanocontainer is straightforward (shown in Figure 1) and enables a separate and controlled modification of the shell's properties, for example, the inner shell can be modified via the copolymerization with functional molecules to introduce, for example, specific binding sites. Furthermore, the crosslink density and thus the mesh size and permeability can be controlled. The outer shell provides the colloidal stability and additional possibility to alter the permeability and can easily be modified via the adsorption of macromolecules on nanoparticles. This can occur in a layer-by-layer fashion [26] or chemically, for example, with binding sites for specific targeting or PEGylation to achieve a stealth effect. An example for a nanogel delivery system of therapeutic siRNA delivery into ovarian carcinomas has been reported by Blackburn et al. [27]: they coupled a peptide mimicking the ligand ephrin-A1 to the surface of approximately $100 \mathrm{~nm}$ diameter core/ shell nanogels harboring high concentrations of siRNA. The peptide ligand binds to its receptor expressed by the tumor cells and permits, therefore, specific targeting and subsequent intracellular delivery of siRNA.

An important aspect of the shell-shell nanocontainers is that two networks are connected. Thus, there is a 'mechanical' coupling of the two shells. In other words, the swelling of the outer and inner networks mutually influence each other. This allows the permeability of the inner shell, which can be influenced in two ways: directly by a stimulus that affects the swelling of the 
polymer network of the inner shell; and indirectly by a stimulus that affects the swelling of the outer shell. The swelling pressure in the outer network will lead to swelling in the outward direction and consequently will stretch the inner network as well.

In our proof-of-concept study, we employed temperature-sensitive polymers. In particular, we used Poly $(N$-isopropylacrylamide) (PNIPAm), which shows a volume phase transition (VPT) in aqueous solution at about $32^{\circ} \mathrm{C}$ and poly $(N$-isopropylmethacrylamide $)$ (PNIPMAm), which shows a similar temperature sensitive behavior in water as PNIPAm, but with a slightly higher VPT temperature of about $42^{\circ} \mathrm{C}$. The VPT temperatures of these polymers can easily be adjusted by incorporation of functional comonomers [28], which can also be used to introduce an additional sensitivity, for example, to $\mathrm{pH}$ [29].

The synthesis of the hollow two-shell nanocontainers followed a straightforward procedure (Figure 1): first, silica nanoparticles were prepared as sacrificial cores. They can be synthesized within a broad range of sizes with very narrow size distributions and can easily be dissolved leading to hollow capsules. Afterward, first a chemically cross-linked shell of PNIPAm was polymerized around the silica core and second a PNIPMAm shell was polymerized around the core-shell nanoparticle. This outer PNIPMAm shell was also cross-linked chemically. Finally, the silica core was dissolved leading to temperature sensitive, hollow two-shell nanocontainers. The structure of these nanocontainers in solution was studied by small-angle neutron scattering employing contrast variation experiments and by computer simulations [25]. Both techniques revealed the unique behavior of the two-shell particles: at intermediate temperatures, the inner polymer network collapses and thus provides a barrier for molecules; the outer shell on the other hand is swollen and provides colloidal stability. The bottom row in Figure 1 corresponds to snapshots of computer simulations, which demonstrate the ability of the nanogels to uptake guest molecules (both shells are swollen), lock and store them in the void (inner and outer shells are collapsed and swollen, respectively) and release the molecules into pure solvent

\section{References}

1 Chen X, Liu L, Jiang Ch. Charge-reversal nanoparticles: novel targeted drug delivery carriers. Acta Pharm. Sin. B 6, 261-267 (2016).

2 Rabišková M, Koziolová E, Jirásková J. Nanoparticulates with drug release based on temperature change. Ceska Slov. Farm. 263, 239-247 (2014).

3 Srinivasan M, Rajabi M, Mousa SA. Multifunctional nanomaterials and their applications in drug upon swelling of the inner shell. It is worth mentioning that both shells consist of uncharged polymers and thus tolerate the presence of salt. Therefore, the hollow twoshell nanocontainers at intermediated temperatures are not sensitive to the ionic strength of the aqueous medium. This makes such nanogels distinctly different from, for example, nanocapsules based on layer-bylayer adsorption of polyelectrolytes due to electrostatic attraction. The physical reason for uptake and release of the neutral molecules by the hollow nanogels has entropic nature.

\section{Future perspective}

Drug nanocarriers that meet the multiple requirements of storage, transport, uptake and release will be able to more specifically reach the goal of targeted drug release. Therefore, multishell and multifunctional nanoparticles have a great potential because their different layers may fulfill distinct needs.

Two-shell hollow nanocontainers have two membranes that can potentially be further functionalized. The two layers can mediate independent functions, which also could be coupled. Our proof-of-concept polymers alter their size and permeability depending on temperature. This feature could be exploited in combination with local hyper- and/or hypothermia therapy. Further possible developments may include coupling of specific ligands as well as introducing specific responsiveness to $\mathrm{pH}$ or osmotic stress. Therefore, two-shell hollow nanocontainers may have great potential to be used as drug delivery carriers for different applications.

\section{Financial \& competing interests disclosure}

The authors thank the Deutsche Forschungsgemeinschaft within the SFB 985 'Functional Microgels and Microgel Systems' and Russian Foundation for Basic Research for financial support. The authors have no other relevant affiliations or financial involvement with any organization or entity with a financial interest in or financial conflict with the subject matter or materials discussed in the manuscript apart from those disclosed.

No writing assistance was utilized in the production of this manuscript.

delivery and cancer therapy. Nanomaterials 5, 1690-1703 (2015).

4 Mura S, Nicolas J, Couvreur P. Stimuli-responsive nanocarriers for drug delivery. Nat. Mater. 12, 991-1003 (2013).

5 Nuhn L, Gietzen S, Mohr K et al. Aggregation behavior of cationic nanohydrogel particles in human blood serum. Biomacromolecules 15, 1526-1533 (2014).

6 Kurtoglu YE, Navath RS, Wang B, Kannan S, Romero R, Kannan RM. Poly(amidoamine) dendrimer-drug conjugates 
with disulfide linkages for intracellular drug delivery. Biomaterials 30, 2112-2121 (2009).

7 Yang YQ, Zheng LS, Guo XD, Qian Y, Zhang LJ. $\mathrm{pH}$-sensitive micelles self-assembled from amphiphilic copolymer brush for delivery of poorly water-soluble drugs. Biomacromolecules 12, 116-122 (2011).

8 Gao GH, Park MJ, Li Y, Im GH et al. The use of pHsensitive positively charged polymeric micelles for protein delivery. Biomaterials 33, 9157-9164 (2012).

9 Cheng Y, Hao J, Lee LA, Biewer MC, Wang Q, Stefan MC. Thermally controlled release of anticancer drug from selfassembled $\gamma$-substituted amphiphilic poly( $\varepsilon$-caprolactone) micellar nanoparticles. Biomacromolecules 13, 2163-2173 (2012).

10 Nuhn L, Hirsch M, Krieg B et al. Cationic nanohydrogel particles as potential siRNA carriers for cellular delivery. ACS Nano 6, 2198-2214 (2012).

11 Smeets NMB, Hoare T. Designing responsive microgels for drug delivery applications. J. Polym. Sci. A-Polym. Chem. 51, 3027-3043 (2013).

12 Mao J, Li Y, Wu T et al. A simple dual-pH responsive prodrug-based polymeric micelles for drug delivery. ACS Appl. Mater. Interfaces 8, 17109-17117 (2016).

13 Shan W, Zhu X, Liu M et al. Overcoming the diffusion barrier of mucus and absorption barrier of epithelium by self-assembled nanoparticles for oral delivery of insulin. ACS Nano 9, 2345-2356 (2015).

14 Swenson CE, Haemmerich D, Maul DH, Knox B, Ehrhart $\mathrm{N}$, Reed RA. Increased duration of heating boosts local drug deposition during radiofrequency ablation in combination with thermally sensitive liposomes (ThermoDox) in a porcine model. PLoS ONE 10(10), e0139752 (2015).

15 Berndt I, Pedersen JS, Richtering W. Temperature-sensitive core-shell microgel particles with dense shell. Angew. Chem. Int. Ed. Engl. 45, 1737 (2006).

16 Keerl M, Pedersen JS, Richtering W. Temperature sensitive copolymer microgels with nanophase separated structure. J. Am. Chem. Soc. 131, 3093-3097 (2009).

17 Kleinen J, Klee A, Richtering W. Influence of architecture on the interaction of negatively charged multisensitive poly $(\mathrm{N}-$ isopropylacrylamide)-co-methacrylic acid microgels with oppositely charged polyelectrolyte: absorption vs adsorption. Langmuir 26, 11258-11265 (2010).

18 Geisel K, Rudov AA, Potemkin II, Richtering W. Hollow and core-shell microgels at oil-water interfaces: spreading of soft particles reduces the compressibility of the monolayer. Langmuir 31, 13145-13154 (2015).

19 Mourran A, Wu Y, Gumerov RA et al. When colloidal particles become polymer coils. Langmuir 32, 723-730 (2016).

20 Gumerov RA, Rumyantsev AM, Rudov AA et al. Mixing of two immiscible liquids within the polymer microgel adsorbed at their interface. ACS Macro Lett. 5, 612-616 (2016).

21 Amstad E, Kim S-H, Weitz DA. Photo- and thermoresponsive polymersomes for triggered release. Angew. Chem. Int. Ed. Engl. 51, 12499-12503 (2012).

22 Stuart MA, Huck WT, Genzer J et al. Emerging applications of stimuli-responsive polymer materials. Nat. Mater. 9, 101-113 (2010).

23 Skorb EV, Möhwald H. 25th anniversary article: dynamic interfaces for responsive encapsulation systems. $A d v$. Mater. 25, 5029-5043 (2013).

24 Dubbert J, Honold T, Pedersen JS et al. How hollow are thermoresponsive hollow nanogels? Macromolecules 47, 8700-8708 (2014).

25 Schmid AJ, Dubbert J, Rudov AA et al. Multi-shell hollow nanogels with responsive shell permeability. Sci. Rep. 6, 22736 (2016).

26 Wong JE, Richtering W. Layer-by-layer assembly on stimuliresponsive microgels. Curr. Opin. Colloid Interface Sci. 13, 403-412 (2008).

27 Blackburn WH, Dickerson EB, Smith MH, McDonald JF, Lyon LA. Peptide-functionalized nanogels for targeted siRNA delivery. Bioconjug. Chem. 20, 960-968 (2009).

28 Keerl M, Smirnovas V, Winter R, Richtering W. Interplay between hydrogen bonding and macromolecular architecture leading to unusual phase behavior in thermosensitive microgels. Angew. Chem. Int. Ed. Engl. 47, 338-341 (2008).

29 Pich A, Richtering W. Microgels by precipitation polymerization: synthesis, characterization, and functionalization. Adv. Polym. Sci. 234, 1-37 (2010). 